\title{
A NOTE ON NILPOTENT ALGEBRAS IN FOUR UNITS*
}

\author{
BY K. S. GHENT
}

1. Introduction. In volume 9 of the Transactions of this Society R. B. Allen gave, without proof, a classification of all associative nilpotent algebras in $n \leqq 4$ units. $\dagger$ These results were later verified by $\mathrm{A}$. A. Albert for $n \leqq 3 . \ddagger$

I have recently completed a reclassification of nilpotent algebras in four units and have discovered several serious errors in Allen's results. Although he stated that his classification separated the algebras into classes of non-equivalent and non-reciprocal algebras, he actually did not accomplish this, as he gave several superfluous cases. Moreover, he erroneously listed certain classes of non-associative algebras which must be replaced by similar classes of associative algebras.

In \$2 I shall prove the validity of the following revision of Allen's 16 classes (labelled A) into my $\$ 9$ classes (labelled G). Allen's classes of irreducible algebras are

$$
\begin{aligned}
& \text { (1A) } e_{1}^{2}=a e_{4}, \quad e_{2} e_{3}=b e_{4}=e_{3}^{2}, \quad e_{3} e_{1}=e_{4}=-e_{3} e_{2}, \\
& \text { (2A) } a e_{1}^{2}=e_{2} e_{1}=e_{2}^{2}=e_{3} e_{2}=b e_{3}^{2}=e_{4}, \\
& \text { (3A) } e_{1}^{2}=e_{3} e_{2}=e_{4}, \\
& \text { (4A) } a e_{1}^{2}=e_{2} e_{1}=e_{2}^{2}=b e_{3}^{2}=e_{4}, \\
& \text { (5A) } a e_{1}^{2}=e_{2}^{2}=b e_{3}^{2}=e_{4}, \\
& \text { (6A) } e_{1}^{2}=e_{3}, \quad e_{2} e_{1}=e_{4}, \quad e_{1} e_{2}=e_{3}+a e_{4}, \quad e_{2}^{2}=b e_{3}+c e_{4}, \\
& \text { (7A) } e_{1}^{2}=e_{3}, \quad e_{2} e_{1}=e_{4}, \quad e_{2}^{2}=a e_{3}+e_{4}, \\
& \text { (8A) } e_{1}^{2}=e_{3}, \quad a e_{2}^{2}=e_{2} e_{1}=e_{4}, \\
& \text { (9A) } e_{1}^{2}=e_{3}, \quad e_{2}^{2}=e_{4}, \\
& \text { (10A) } e_{1}^{2}=e_{3}, \quad e_{1} e_{2}=a e_{2} e_{1}=e_{2}^{2}=e_{4}, \\
& \text { (11A) } e_{1}^{2}=e_{3}, \quad e_{1} e_{2}=a e_{2} e_{1}=e_{4}, \\
& \text { (12A) } e_{1}^{2}=a e_{2}^{2}=e_{3}, \quad e_{2} e_{1}=a e_{1} e_{2}=e_{4},
\end{aligned}
$$

* Presented to the Society, October 28, 1933.

$\dagger$ Transactions of this Society, vol. 9, pp. 213.

$\ddagger$ In his master thesis, pp. 5-7.

$\S$ As may be observed, my own classes are but minor revisions of Allen's classes. These revisions are necessary in order that all algebras may be included. 
(13A) $e_{1}^{2}=e_{3}, \quad e_{2}^{2}=a e_{3}+e_{4}, \quad e_{1} e_{2}=a e_{4}, \quad e_{2} e_{1}=e_{4}$,

(14A) $e_{1}^{2}=e_{3}, \quad e_{1} e_{3}=a e_{2} e_{1}=e_{2}^{2}=e_{3}^{2}=e_{4}$,

(15A) $e_{1}^{2}=e_{3}, \quad e_{1} e_{3}=e_{4}=e_{2} e_{1}$,

(16A) $e_{i}=e_{1}{ }^{i}$,

with constants $a, b, c$ in the reference field $F$, while my own classes are

(1G) Same as (1A),

(4G) $e_{2} e_{1}=e_{2}^{2}=e_{4}, \quad e_{1}^{2}=a e_{4}, \quad e_{3}^{2}=b e_{4}$,

(5G) $e_{2}^{2}=e_{4}, \quad e_{1}^{2}=a e_{4}, \quad e_{3}^{2}=b e_{4}$,

(6G) Same as (6A),

(9G) Same as (9A),

(12G) $e_{1}^{2}=e_{3}, \quad e_{2}^{2}=a e_{3}, \quad e_{1} e_{2}=e_{2} e_{1}=e_{4}$,

(14G) $\quad e_{1}^{2}=e_{3}, \quad e_{1} e_{3}=e_{3} e_{1}=e_{2} e_{1}=e_{4}, \quad e_{2}^{2}=a e_{4}$,

(15G) $e_{1}^{2}=e_{3}, \quad e_{1} e_{3}=e_{3} e_{1}=e_{4}, \quad e_{2}^{2}=a e_{4}$,

(16G) Same as (16A).

2. The Revision. The classes (14A) and (15A) obviously do not belong in a table of associative algebras. For, if $e_{1}^{2}=e_{3}$, then $e_{1}^{3}=e_{1} e_{3}=e_{3} e_{1}$. Hence, if $e_{1} e_{3} \neq 0$, then $e_{3} e_{1} \neq 0$ and further $e_{3}^{2}=e_{1}^{4}=e_{1} e_{1}^{3}=e_{1}\left(e_{1} e_{3}\right)=e_{1} a_{13} e_{4}=0$. Both of these conclusions contradict the tables numbered (14A) and (15A).

Class (3A) is superfluous and may be shown to be a sub-case of class $(4 G)$. For in class (3A) we have

$$
e_{1}^{2}=e_{3} e_{2}=e_{4},
$$

or, on replacing $e_{3}$ by $e_{2}, e_{2}$ by $e_{1}$, and $e_{1}$ by $e_{3}$,

$$
e_{3}^{2}=e_{2} e_{1}=e_{4} \text {. }
$$

Put $e_{2}^{\prime}=e_{1}+e_{2}$ and obtain

$$
e_{2}^{\prime 2}=e_{2}^{\prime} e_{1}=e_{3}^{2}=e_{4},
$$

which is class (4G) for $a=0$ and $b=1$.

Class (7A) is a special case of class (6A). For in class (7A)

$$
e_{1}^{2}=e_{3}, \quad e_{2} e_{1}=e_{4}, \quad e_{2}^{2}=e_{4}+d e_{3} .
$$

Put $e_{2}^{\prime}=e_{1}+e_{2}$ and obtain

(5) $\left\{\begin{array}{l}e_{1}^{2}=e_{3}, \quad e_{1} e_{2}^{\prime}=e_{1}^{2}+e_{1} e_{2}=e_{3}, \quad e_{2}^{\prime} e_{1}=e_{1}^{2}+e_{2} e_{1}=e_{3}+e_{4}, \\ e_{2}^{\prime 2}=e_{1}^{2}+e_{1} e_{2}+e_{2} e_{1}+e_{2}^{2}=2 e_{4}+(d+1) e_{3} .\end{array}\right.$ 
Let $\left(e_{3}+e_{4}\right)=e_{4}^{\prime}$, so that

(6) $e_{1}^{2}=e_{3}, \quad e_{2}^{\prime} e_{1}=e_{4}^{\prime}, \quad e_{1} e_{2}^{\prime}=e_{3}, \quad e_{2}^{\prime 2}=(d-1) e_{3}+2 e_{4}^{\prime}$,

which is class (6A) for $a=0, b=d-1$, and $c=2$.

We may also omit class (8A). Since $e_{4}$ is a basal unit, $a \neq 0$. Then if $b=(1 / a)$, class (8A) becomes

$$
e_{1}^{2}=e_{3}, \quad e_{2} e_{1}=e_{4}, \quad e_{2}^{2}=b e_{4} .
$$

Let $b e_{4}=e_{4}^{\prime}$ and put $e_{1}=\left(e_{1}^{\prime} / b\right)$ and let $b^{2} e_{3}=e_{3}^{\prime}$, so that

$$
e_{2}^{2}=e_{2} e_{1}^{\prime}=e_{4}^{\prime}, \quad e_{1}^{\prime 2}=e_{3}^{\prime} .
$$

Replace $e_{2}$ by $e_{2}^{\prime}=\left(e_{1}^{\prime}+e_{2}\right)$ to give

(9) $e_{1}^{\prime 2}=e_{3}^{\prime}, \quad e_{1}^{\prime} e_{2}^{\prime}=e_{3}^{\prime}, \quad e_{2}^{\prime} e_{1}^{\prime}=e_{3}^{\prime}+e_{4}^{\prime}, \quad e_{2}^{\prime 2}=e_{3}^{\prime}+2 e_{4}^{\prime}$.

Let $\left(e_{3}^{\prime}+e_{4}^{\prime}\right)=e_{4}^{\prime \prime}$ and obtain

(10) $e_{1}^{\prime 2}=e_{3}^{\prime}, \quad e_{1}^{\prime} e_{2}^{\prime}=e_{3}^{\prime}, \quad e_{2}^{\prime} e_{1}^{\prime}=e_{4}^{\prime \prime}, e_{2}^{\prime 2}=-e_{3}^{\prime}+2 e_{4}^{\prime}$,

which is class (6A) for $a=0, b=-1$ and $c=2$.

Class (10A) likewise involves no new algebras. Algebras (10A) have the form

$$
e_{1}^{2}=e_{3}, \quad e_{1} e_{2}=a e_{2} e_{1}=e_{2}^{2}=e_{4},
$$

and again $a \neq 0$, since $e_{4}$ is a basal unit. If $a=1$, replace $e_{1}$ by $e_{1}^{\prime}=e_{1}-e_{2}$ and let $\left(e_{3}-e_{4}\right)=e_{3}^{\prime}$, so that

$$
e_{1}^{\prime 2}=e_{3}^{\prime}, \quad e_{2}^{2}=e_{4},
$$

while all other products vanish. This is class (9A).

Henceforth, in class (10A) assume $a \neq 0,1$ and let $b=1 / a$, so that algebras (10A) become

$$
e_{1}^{2}=e_{3}, \quad e_{1} e_{2}=e_{2}^{2}=e_{4}, \quad e_{2} e_{1}=b e_{4}, \quad(b \neq 0,1) .
$$

Let $\left(e_{1}+e_{2}\right) r=e_{2}^{\prime}$, where $r=b /(b-1)$; then

$$
\left\{\begin{aligned}
e_{1} e_{2}^{\prime} & =r\left(e_{3}+e_{4}\right), \quad e_{2}^{\prime} e_{1}=r\left(e_{3}+b e_{4}\right), \\
e_{2}^{\prime 2} & =r^{2}\left(e_{3}+e_{4}+b e_{4}+e_{4}\right), \quad e_{1}^{2}=e_{3} .
\end{aligned}\right.
$$

Let $r\left(e_{3}+b e_{4}\right)=e_{4}^{\prime} ;$ then $e_{4}=\left(e_{4}^{\prime}-r e_{3}\right) /(b r)$. Hence (14) becomes 


$$
\begin{aligned}
e_{1}^{2} & =e_{3}, \quad e_{2}^{\prime} e_{1}=e_{4}^{\prime}, \\
e_{1} e_{2}^{\prime} & =r e_{3}+\frac{r\left(e_{4}^{\prime}-r e_{3}\right)}{r b}=r e_{3}\left(1-\frac{1}{b}\right)+\frac{e_{4}^{\prime}}{b}=e_{3}+\frac{e_{4}^{\prime}}{b}, \\
e_{2}^{\prime 2} & =A e_{3}+B e_{4}, \quad(A \text { and } B \text { in } F) .
\end{aligned}
$$

This is evidently class (6A). Thus we have shown that algebras (10A) are transformable either into algebras (6A), or into algebras (9A).

The class of algebras (11A) likewise contains only algebras that are included in other classes. Algebras (11A) are of the form

$$
e_{1}^{2}=e_{3}, \quad e_{1} e_{2}=a e_{2} e_{1}=e_{4},
$$

and $a \neq 0$, since $e_{4}$ is a basal unit. If $a=1,(16)$ represents commutative algebras which are included in my class $(12 \mathrm{G})$ for $a=0$.

Henceforth in class (11A), assume $a \neq 0,1$. Algebras (11A) become

$$
e_{1}^{2}=e_{3}, \quad e_{1} e_{2}=e_{4}, \quad e_{2} e_{1}=b e_{4}, \quad(b=1 / a) .
$$

Put $\left(e_{1}+e_{2}\right) r=e_{2}^{\prime}$, where $r=b /(b-1)$. Then

$$
e_{2}^{\prime} e_{1}=r\left(e_{3}+b e_{4}\right), \quad e_{1} e_{2}^{\prime}=r\left(e_{3}+e_{4}\right) \text {. }
$$

Let $r\left(e_{3}+b e_{4}\right)=e_{4}^{\prime}$. Then $e_{4}=\left(e_{4}^{\prime}-r e_{3}\right) /(b r)$ and the algebras are of the form

$$
\begin{gathered}
e_{1}^{2}=e_{3}, \quad e_{2} e_{1}=e_{4}, \quad e_{2}^{2}=a e_{3}+B e_{4}, \\
e_{1} e_{2}=r e_{3}+\frac{r\left(e_{4}^{\prime}-r e_{3}\right)}{b r}=\frac{r(b-1) e_{3}}{b}+\frac{e_{4}^{\prime}}{b}=e_{3}+\frac{e_{4}^{\prime}}{b}
\end{gathered}
$$

which is class (6A). Hence algebras (11A) include only algebras contained in other classes.

Let us next examine algebras (12A) which are of the form

$$
e_{1}^{2}=a e_{2}^{2}=e_{3}, \quad e_{2} e_{1}=a e_{1} e_{2}=e_{4},
$$

and $a \neq 0$, since $e_{3}$ and $e_{4}$ are basal units. If $a=1$, we have class $(12 \mathrm{G})$ for $a=1$ of my revised table. Henceforth assume $a \neq 0,1$. Class (12A) then has the form

(21) $e_{1}^{2}=e_{3}, e_{2}^{2}=A e_{3}, e_{2} e_{1}=e_{4}, e_{1} e_{2}=A e_{4}, \quad(A=1 / a \neq 1,0)$.

Put $e_{2}^{\prime}=r\left(e_{1}+e_{2}\right)$, where $r=1 /(1-A)$, so that 


$$
e_{1} e_{2}^{\prime}=r\left(e_{3}+A e_{4}\right) \text {, and } e_{2}^{\prime} e_{1}=r\left(e_{3}+e_{4}\right) \text {. }
$$

Let $\left(e_{3}+e_{4}\right) r=e_{4}^{\prime}$, and then $e_{4}=\left(e_{4}^{\prime}-r e_{3}\right) / r$ and

$$
\begin{aligned}
e_{1}^{2} & =e_{3}, \quad e_{2} e_{1}=e_{4}^{\prime}, \quad e_{2}^{2}=B e_{3}+D e_{4}, \\
e_{1} e_{2} & =r e_{3}+\frac{r A\left(e_{4}^{\prime}-r e_{3}\right)}{r}=e_{3}+A e_{4}^{\prime},
\end{aligned}
$$

which is class (6A). Hence class (12A) as given by Allen is superfluous except when $a=1$.

Class (13A) can be shown unnecessary. It has the form

$$
e_{1}^{2}=e_{3}, \quad e_{2}^{2}=a e_{3}+e_{4}, \quad e_{1} e_{2}=a e_{4}, \quad e_{2} e_{1}=e_{4} \text {. }
$$

If $a=0$, the algebras (24) are easily transformable to class (6A) (see algebras of class (8A) above). If $a=1$,

$$
e_{1}^{2}=e_{3}, \quad e_{2}^{2}=e_{3}+e_{4}, \quad e_{1} e_{2}=e_{2} e_{1}=e_{4} .
$$

Put $e_{2}^{\prime}=e_{1}-2 e_{2}$ and let $e_{3}-2 e_{4}=e_{4}^{\prime}$, so that

$$
\begin{aligned}
e_{1}^{2}= & e_{3}, \quad e_{2}^{\prime 2}=e_{1}^{2}-2 e_{1} e_{2}-2 e_{2} e_{1}+4 e_{2}^{2}=e_{3}-2 e_{4}-2 e_{4} \\
+4 e_{4}+4 e_{3} & =5 e_{3}, \quad e_{1} e_{2}^{\prime}=e_{2}^{\prime} e_{1}=e_{3}-2 e_{4}=e_{4}^{\prime},
\end{aligned}
$$

which is class $(12 \mathrm{G})$.

Henceforth in class (13A), assume $a \neq 0,1$. Put $e_{1}^{\prime}=e_{1}+e_{2}$ and $e_{2}^{\prime}=r e_{2}$, where $r=1 /\left(a^{2}-a\right)$. Then

$$
\begin{aligned}
e_{1}^{\prime 2} & =e_{1}^{2}+e_{1} e_{2}+e_{2} e_{1}+e_{2}^{2}=e_{3}(1+a)+e_{4}(2+a), \\
e_{1}^{\prime} e_{2}^{\prime} & =r a e_{3}+r(a+1) e_{4}, \quad e_{2}^{\prime} e_{1}^{\prime}=2 r e_{4}+a r e_{3}, \\
e_{2}^{\prime 2} & =r^{2} a e_{3}+r^{2} e_{4} .
\end{aligned}
$$

Pass to the reciprocal algebra and let

$$
\left(e_{3}(1+a)+e_{4}(2+a)\right)=e_{3}^{\prime} \text { and }\left(r a e_{3}+r(a+1) e_{4}\right)=e_{4}^{\prime} \text {. }
$$

Then

and similarly

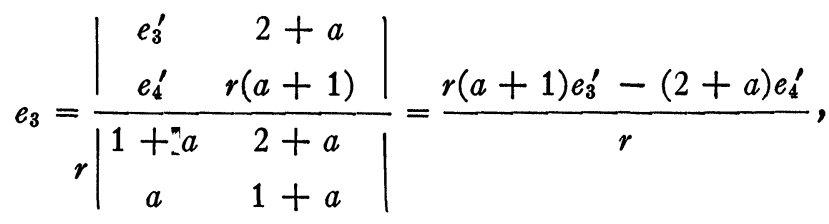

$$
e_{4}=\frac{e_{4}^{\prime}(1+a)-e_{8}^{\prime} r a}{r}
$$


Hence our algebras have the form

$$
\begin{aligned}
e_{1}^{2} & =e_{3}^{\prime}, \quad e_{2} e_{1}=e_{4}^{\prime} \\
e_{1} e_{2} & =\frac{2 r\left(e_{4}^{\prime}(1+a)-e_{3}^{\prime} r a\right)}{r}+\frac{a r\left(r(a+1) e_{3}^{\prime}-(2+a) e_{4}^{\prime}\right)}{r} \\
& =r e_{3}^{\prime}\left(a^{2}-a\right)+e_{4}^{\prime}\left(2-a^{2}\right)=e_{3}^{\prime}+e_{4}^{\prime}\left(2-a^{2}\right), \\
e_{2}^{2} & =B e_{3}^{\prime}+D e_{4}^{\prime}, \quad(B \text { and } D \text { in } F) .
\end{aligned}
$$

These are again algebras (6A). We have now shown that class (13A) contains only algebras contained in class $(6 \mathrm{~A})$ or in class (12G).

Class (2A) is also superfluous, as it can be transformed either into class (1A) or into class (4G) as follows. Since $e_{4}$ is a basal unit, $a \neq 0$, and $b \neq 0$. Hence class (2A) has the form

$$
e_{1}^{2}=a_{1} e_{3}, \quad e_{2}^{2}=e_{2} e_{1}=e_{3} e_{2}=e_{4}, \quad e_{3}^{2}=b_{1} e_{4} .
$$

If $a_{1}=b_{1}=0$, replace $e_{3}$ by $e_{3}^{\prime}=e_{1}+e_{3}$ to give

$$
e_{2}^{2}=e_{2} e_{1}=e_{3} e_{2}=e_{2} e_{3}=e_{4},
$$

or, on interchanging $e_{3}$ and $e_{2}$,

$$
e_{3}^{2}=e_{2} e_{3}=e_{4}, \quad e_{3} e_{1}=e_{4}=e_{3} e_{2} .
$$

Put $e_{1}^{\prime}=-e_{1}$ and $-e_{4}=e_{4}^{\prime}$ and obtain class (1A) for $a=0$ and $b=-1$.

Now assume $a_{1} \neq 0, b_{1} \neq 0, a_{1} \neq-b_{1}$. Then the non-singular transformation

$$
e_{1}^{\prime}=-\frac{1}{a_{1}} e_{1}+e_{2}, \quad e_{2}^{\prime}=-\frac{b_{1}}{a_{1}} e_{1}+e_{3}, \quad e_{3}^{\prime}=e_{1}+e_{3}
$$

yields algebras of the form (33) when applied to class (2A):

$$
e_{1}^{\prime 2}=A e_{4}^{\prime}, \quad e_{2}^{\prime 2}=e_{4}^{\prime}, \quad e_{3}^{\prime 2}=B e_{4}^{\prime}, \quad e_{2}^{\prime} \epsilon_{1}^{\prime}=M e_{4}^{\prime},
$$

where $e_{4}^{\prime}=\left(b_{1}^{2} / a_{1}-b_{1}\right) e_{4}$ and $A, B$ and $M$ are easily calculated numbers of the field $F$. The form (33) is readily transformable to class $(4 \mathrm{G})$.

If $a_{1} \neq 0, b_{1}=0$, the above transformation (32) gives

$$
e_{1}^{\prime 2}=e_{4}, \quad e_{3}^{\prime 2}=a_{1} e_{4}, \quad e_{2}^{\prime} e_{1}^{\prime}=e_{4},
$$

or, interchanging $e_{1}^{\prime}$ and $e_{2}^{\prime}$, 


$$
e_{2}^{\prime 2}=e_{4}, \quad e_{3}^{\prime 2}=a_{1} e_{4}, \quad e_{1}^{\prime} e_{2}^{\prime}=e_{4} .
$$

Passing to the reciprocal algebra, we have class $(4 \mathrm{G})$ for $a_{1}=0$.

If $a_{1}=0$ and $b_{1} \neq 0$, interchanging $e_{1}$ and $e_{3}$ and passing to the reciprocal algebra, we again have the case $a_{1} \neq 0, b_{1}=0$. If $a_{1}=-b_{1}$ the transformation (32) is singular. Transformation (36), however, can be used to transform class (2A) with $a_{1}=-b_{1}$ into class (2A) for $a_{1} \neq-b_{1}$, unless $a_{1}{ }^{2}=2$ :

$$
\text { (36) } \begin{aligned}
e_{1}^{\prime} & =-2 e_{1}+e_{2}-2 e_{3}, \\
e_{3}^{\prime} & =e_{1}+e_{2}+e_{3} .
\end{aligned}
$$

This non-singular transformation gives, when applied to class (2A) for $a_{1}=-b_{1}$,

$$
\begin{aligned}
e_{1}^{\prime 2} & =-3 e_{4}, \quad e_{3}^{\prime 2}=3 e_{4}, \quad e_{2}^{\prime 2}=\frac{9 a_{1}{ }^{2}}{4} e_{4}, \\
e_{2}^{\prime} e_{1}^{\prime} & =\frac{3}{2} e_{4}, \quad e_{3}^{\prime} e_{2}^{\prime}=\frac{3 a_{1}^{2}-3}{2} e_{4} .
\end{aligned}
$$

Let us set $e_{1}^{\prime}=(3 / 2)\left(4 /\left(9 a_{1}^{2}\right)\right) e_{1}^{\prime \prime}$, so that $e_{2}^{\prime} e_{1}^{\prime \prime}=\left(9 a_{1}^{2} / 4\right) e_{4}$ and $e_{1}^{\prime \prime 2}=-(27 / 4) a_{1}^{4} e_{4}$. Let $e_{3}^{\prime}=\left[\left(3 a_{1}^{2}-3\right) / 2\right]\left[4 /\left(9 a_{1}^{2}\right)\right] e_{3}^{\prime \prime}$, so that $e_{3}^{\prime \prime} e_{2}^{\prime}=\left(9 a_{1}^{2} / 4\right) e_{4}$ and $e_{3}^{\prime 2}=\left[\left(2\left(a_{1}^{2}-1\right) /\left(3 a_{1}^{2}\right)\right)^{2}(3)\right] e_{4}$. If we set $\left(9 a_{1}^{2} / 4\right) e_{4}=e_{4}{ }^{\prime}$, we obtain

$$
e_{2}^{\prime 2}=e_{3}^{\prime \prime} e_{2}^{\prime}=e_{2}^{\prime} e_{1}^{\prime \prime}=e_{4}^{\prime}, \quad e_{1}^{\prime \prime 2}=A e_{4}^{\prime}, \quad e_{3}^{\prime \prime 2}=B e_{4}^{\prime},
$$

where $A \neq-B$. Hence we can now use (32) to carry algebras (2A) into algebras (4A).

Assume now for class (2A), $a_{1}=-b_{1}$ and $a_{1}{ }^{2}=b_{1}{ }^{2}=2$ and apply

$$
\begin{aligned}
& e_{1}^{\prime}=\left(-a_{1}-1\right) e_{1}+e_{2}+\left(-a_{1}-1\right) e_{3}, \\
& e_{2}^{\prime}=2 a_{1}\left(a_{1}+1\right) e_{1}-\left(2 a_{1}^{2}+a_{1}-1\right) e_{2}+\left(2 a_{1}^{2}-1\right) e_{3}, \\
& e_{3}^{\prime}=a_{1} e_{1}+e_{2}+a_{1} e_{3} .
\end{aligned}
$$

This non-singular transformation gives 


$$
\begin{aligned}
e_{1}^{\prime 2} & =\left(-2 a_{1}-1\right) e_{4}, \\
e_{2}^{\prime 2} & =\left(4 a_{1}^{5}+8 a_{1}^{4}-3 a_{1}^{2}+a_{1}+1\right) e_{4}=A \epsilon_{4}, \\
e_{3}^{\prime 2} & =\left(2 a_{1}+1\right) e_{4}, \quad e_{2}^{\prime} e_{1}^{\prime}=\left(-2 a_{1}\right) e_{4}, \\
e_{3}^{\prime} e_{2}^{\prime} & =\left(2 a_{1}+1\right) e_{4}, \quad e_{1}^{\prime} e_{3}^{\prime}=e_{3}^{\prime} e_{1}^{\prime}=e_{2}^{\prime} e_{3}^{\prime}=e_{1}^{\prime} e_{2}^{\prime}=0 .
\end{aligned}
$$

In (40), $a_{1}=2^{1 / 2}$ or $a_{1}=-2^{1 / 2}$ does not cause any of the parameters to vanish nor does it make the transformation (39) singular. Let $e_{1}^{\prime}=\left(-2 a_{1} / A\right) e_{1}^{\prime \prime}, e_{3}^{\prime}=\left[\left(2 a_{1}+1\right) / A\right] e_{3}^{\prime \prime}, A e_{4}=e_{4}^{\prime}$, so that

$$
\begin{aligned}
e_{2}^{\prime} e_{1}^{\prime \prime} & =e_{3}^{\prime \prime} e_{2}^{\prime}=e_{2}^{\prime 2}=e_{4}^{\prime}, \quad e_{1}^{\prime \prime 2}=\frac{A^{2}\left(-2 a_{1}-1\right)}{\left(2 a_{1}\right)^{2}} \frac{e_{4}^{\prime}}{A}=M e_{4}^{\prime}, \\
e_{3}^{\prime \prime 2} & =\frac{A^{2}\left(2 a_{1}+1\right)}{\left(2 a_{1}+1\right)^{2} A} e_{4}^{\prime}=N e_{4}^{\prime},
\end{aligned}
$$

where it is quite obvious that $M \neq-N$. We can now use (32) to carry algebras (2A) into algebras (4G).

We have thus shown that classes (2A), (3A), (7A), (8A), $(10 \mathrm{~A}),(11 \mathrm{~A})$, and $(13 \mathrm{~A})$ are superfluous and can be omitted. Classes (14A) and (15A) are erroneous and have been replaced by classes $(14 \mathrm{G})$ and $(15 \mathrm{G})$ which arise naturally in the process of classification. Classes (4A), (5A), and (12A) have been altered so as to allow the parameters to take the value zero which is necessary if all algebras are to be included. The classes of algegras, in my table, with the exception of (1G), (4G), (9G), and $(12 \mathrm{G})$, are non-equivalent and non-reciprocal regardless of the field $F$ or special values of the constants in the algebras. Algebras of $(12 \mathrm{G})$ are equivalent to algebras of (9G) if and only if $a$ is the square of a number of $F$. Algebras in classes $(1 \mathrm{G})$ and (4G) also may be equivalent if the field and values of $a$ and $b$ are such that certain equations given in my master dissertation are satisfied.

The University of Chicago 